\title{
Ischemic Mitral Regurgitation
}

\author{
- Where Do We Stand? -
}

Jack H. Boyd, MD

\begin{abstract}
Chronic ischemic mitral regurgitation (IMR) is still a significant clinical problem. It is present in $10-20 \%$ of patients with coronary artery disease and is associated with a worse prognosis after myocardial infarction and subsequent revascularization. Currently, coronary artery bypass grafting combined with restrictive annuloplasty is the most commonly performed surgical procedure, although novel approaches have been used in limited numbers with varying degrees of success. The purpose of this review is to clarify the rationale for the surgical techniques applicable to IMR. In order to do so, the condition will be defined and the evolution of classic or traditional surgical approaches to repairing or replacing the mitral valve in the setting of IMR will be described. Finally, novel approaches to the repair of the ischemic mitral valve will be considered. (Circ J 2013; 77: 1952-1956)
\end{abstract}

Key Words: Mitral valve; Surgery; Valvular disorders; Ventricular remodeling

C hronic ischemic mitral regurgitation (IMR) remains a significant clinical problem. It is present in 10-20\% of patients with coronary artery disease and is associated with a worse prognosis after myocardial infarction and subsequent revascularization. ${ }^{1}$ Patients with IMR have a worse prognosis than those with coronary artery disease alone, and this detriment in prognosis becomes greater with increasing severity of regurgitation. ${ }^{2}$ Medical therapy is limited in its efficacy. A combination of angiotensin-converting enzyme inhibitors and beta-blockade reverses negative left ventricular remodeling, yet a decrease in the incidence or severity of IMR has not been demonstrated by this approach. ${ }^{3}$ Current surgical treatment strategies also have room for improvement. The persistence and recurrence rates of mitral regurgitation remain high in patients undergoing restrictive annuloplasty and coronary artery bypass grafting (CABG). No survival benefit has been demonstrated at 10 years compared with $\mathrm{CABG}$ alone. ${ }^{4}$ Indications for surgery in this group are inadequately defined and based on general consensus. Surgery performed for IMR ranges from $\mathrm{CABG}$ alone to $\mathrm{CABG}$ and mitral valve surgery. Mitral valve surgery encompasses annuloplasty, complex repairs, and replacement. Variation in the type of surgery performed and inconsistencies of technique within each type of surgery confound conclusions drawn from many previous studies on this topic. Furthermore, many of the surgical techniques applied when repairing the mitral valve in the setting of coronary ischemia are borrowed from techniques developed to repair the degenerative mitral valve. An attempt to distinguish chronic IMR from other forms of mitral regurgitation on the basis of pathophysiologic mechanisms is a step toward improving its treatment. By examining historical and current surgical approaches to this disease, it may be possible to better elucidate successful repair strategies and target future improvements.

\section{Definition}

Chronic IMR is mitral regurgitation post infarction or an ischemia-induced wall motion abnormality. Subsequent ventricular remodeling displaces the papillary muscles towards the ventricular apex, which, in turn, draws the fixed length chordae tendineae away from the line of coaptation. The retracted, not foreshortened, chordae tendineae tether the valve leaflets, preventing normal valve closure and resulting in valvular incompetence. The leaflets appear tethered but are otherwise normal (Figure 1).3,4

Clinically, this will be apparent with evidence of prior myocardial infarction by typical ECG findings and/or wall motion abnormalities on echocardiography. Appropriately correlative findings on cardiac catheterization or other appropriate imaging technique confirm the ischemic etiology. Echocardiography will demonstrate the tethering of the leaflets predominantly in the P2 and P3 segments of the posterior leaflet and the resultant regurgitant jet. No evidence of organic mitral valve disease should be present.

\section{Evolution of Surgical Approaches}

Initially, the surgical approach to severe IMR was to replace the valve, which restored the competency of the valve, but short- and long-term mortality rates were unacceptably high. Excision of the subvalvular apparatus during replacement led to a loss of normal ventricular geometry, which subsequently impaired ventricular function, negatively affecting survival. From this realization, efforts were then directed at maintaining

Received June 12, 2013; accepted June 13, 2013; released online July 19, 2013

Division of Cardiothoracic Surgery, Department of Surgery, Indiana University School of Medicine, Indianapolis, IN, USA

Mailing address: Jack H. Boyd, MD, Assistant Professor of Surgery, Division of Cardiothoracic Surgery, Department of Surgery, Indiana

University School of Medicine, 1801 N. Senate Blvd, MPC-2, Suite 3550, Indianapolis, IN 46202, USA. E-mail: jhboyd@iupui.edu

ISSN-1346-9843 doi:10.1253/circj.CJ-13-0743

All rights are reserved to the Japanese Circulation Society. For permissions, please e-mail: cj@j-circ.or.jp 
ventricular function while restoring valvular competency in patients undergoing surgery for IMR.

These efforts took 2 directions. On one hand, it was found that by preserving a significant portion of the subvalvular apparatus during mitral valve replacement, normal left ventricular geometry could be better preserved. Ventricular function was better maintained and survival improved. ${ }^{5}$ On the other hand, mitral valve repair techniques were developed to compensate for the shortcomings of valve replacement. Valve repair techniques preserve the subvalvular apparatus and consequently left ventricular systolic function is better preserved than after valve replacement. ${ }^{6}$ Repair has the advantages of resistance to infection and better durability than bioprosthetics, and avoids the need for chronic anticoagulation.

During this time, the concept of restrictive annuloplasty was introduced. Bach and Bolling ${ }^{7}$ treated 13 patients with end-stage dilated cardiomyopathies and severe mitral regurgitation (only 4 had an ischemic etiology) with a restrictive annuloplasty. It was hypothesized that reducing the size of the mitral annulus would increase the amount of leaflet coaptation and decrease the amount of regurgitation while maintaining ventricular function. In all of the patients studied an improvement in symptoms was noted. The technique is relatively simple to perform and is reproducible. Consequently, it has become the most frequently used technique in the surgical treatment of IMR.

More recently, the efficacy of this technique has been questioned: follow-up is frequently incomplete, technique is inconsistent, and the cohorts are poorly defined or heterogeneous. ${ }^{3}$ One of the major shortcomings of this approach is the high incidence of late failures. A restrictive annuloplasty addresses Type I mitral regurgitation directly, and may compensate for Type IIIb regurgitation in some cases. It does not, however, address the ventricular remodeling and papillary muscle displacement, which leads to leaflet tenting.

Restrictive annuloplasty compensates appropriately and adequately for the annular dilatation of Type I dysfunction, but is inadequate for the treatment of Type IIIb dysfunction be-

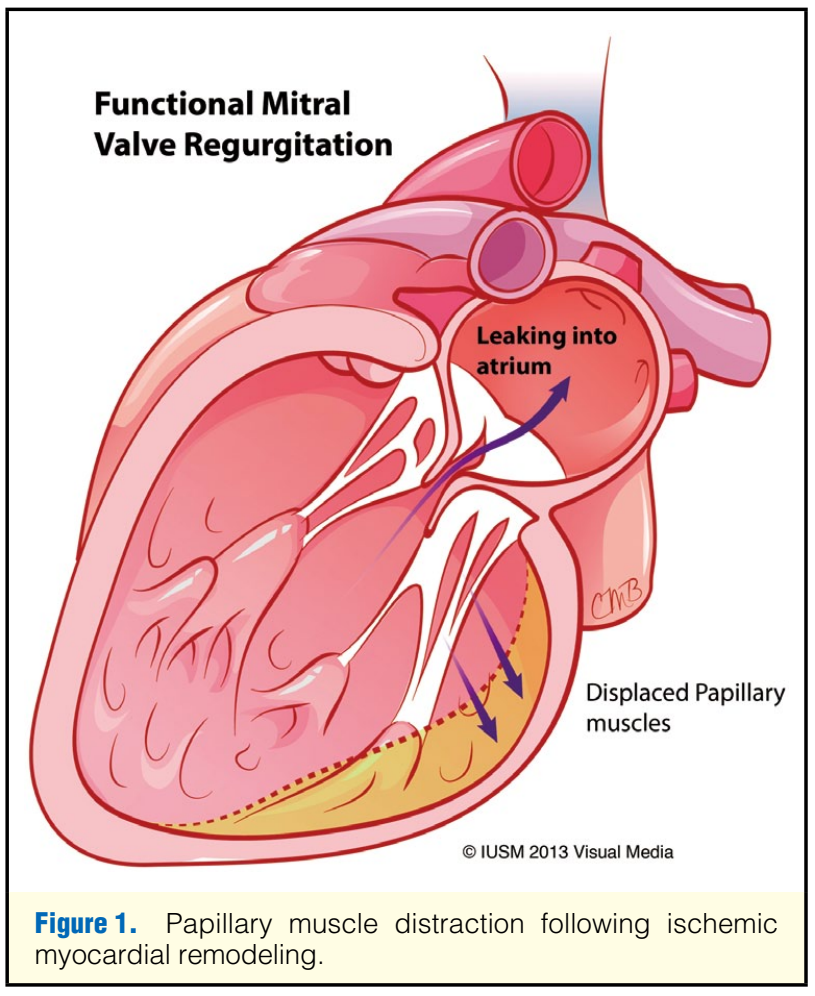

cause of the failure to address the pathology directly. A comprehensive approach to the repair of the ischemic mitral valve must take into account all aspects of the disease process. In addition to the etiology of coronary ischemia, secondary lesions in IMR (ie, ventricular dilatation, papillary muscle displacement, chordal tethering, and leaflet restriction) should also be addressed. Once accomplished, the Type IIIb dysfunction of IMR will be ameliorated.

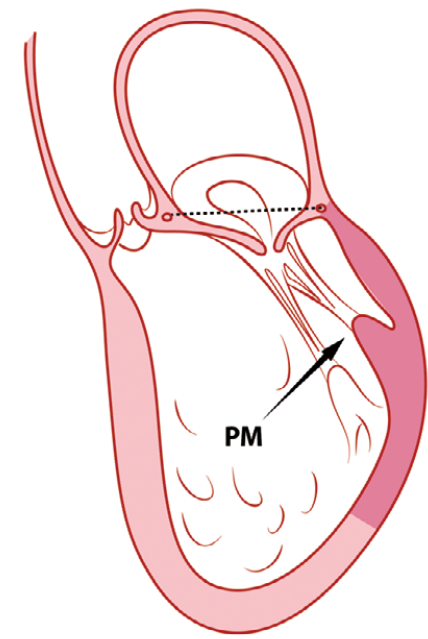

Inferior MI

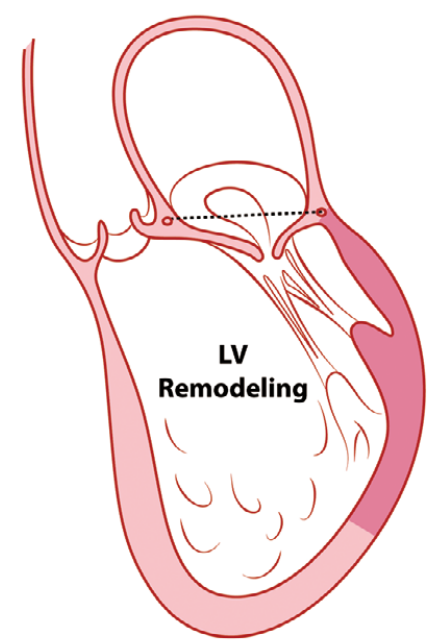

Chronic MI

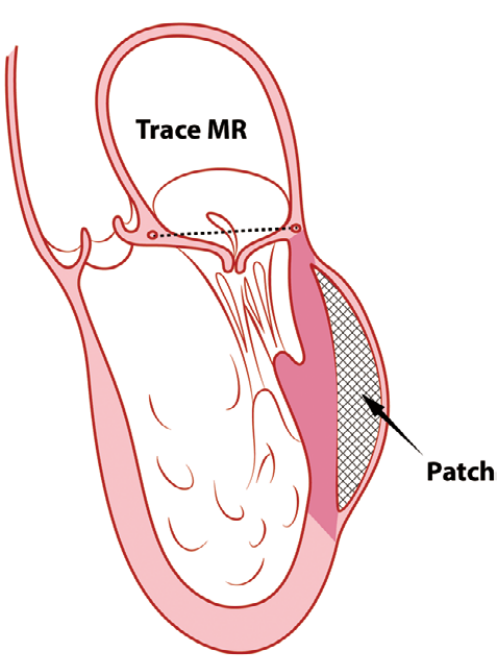

Patch

Figure 2. Epicardial balloon placement counteracts papillary muscle (PM) displacement. LV, left ventricle; MI, myocardial infarction; MR, mitral regurgitation. 


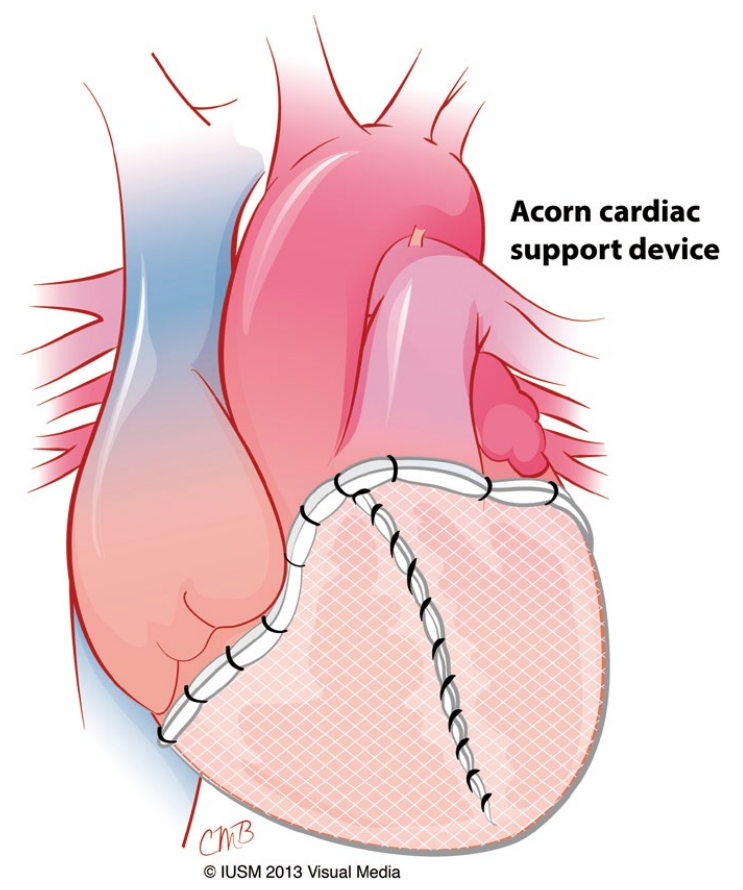

Figure 3. Corcap Cardiac Support Device.

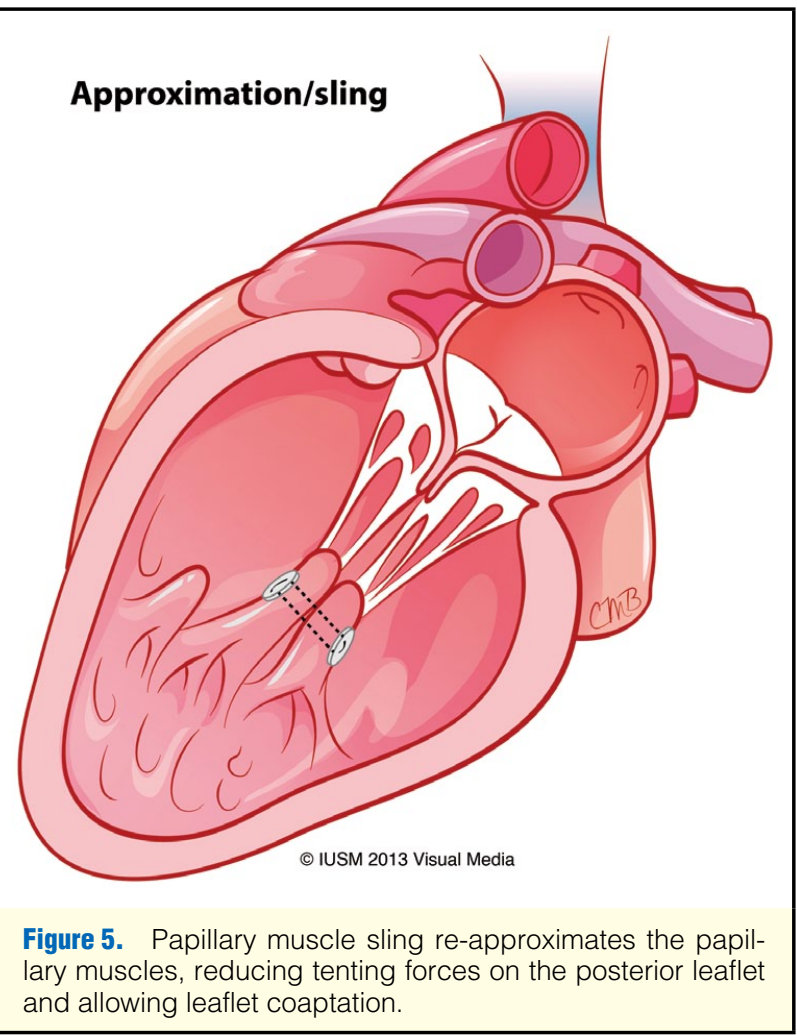

\section{Novel Approaches to Repair of the Ischemic Mitral Valve}

The ideal surgical repair of the regurgitant ischemic mitral

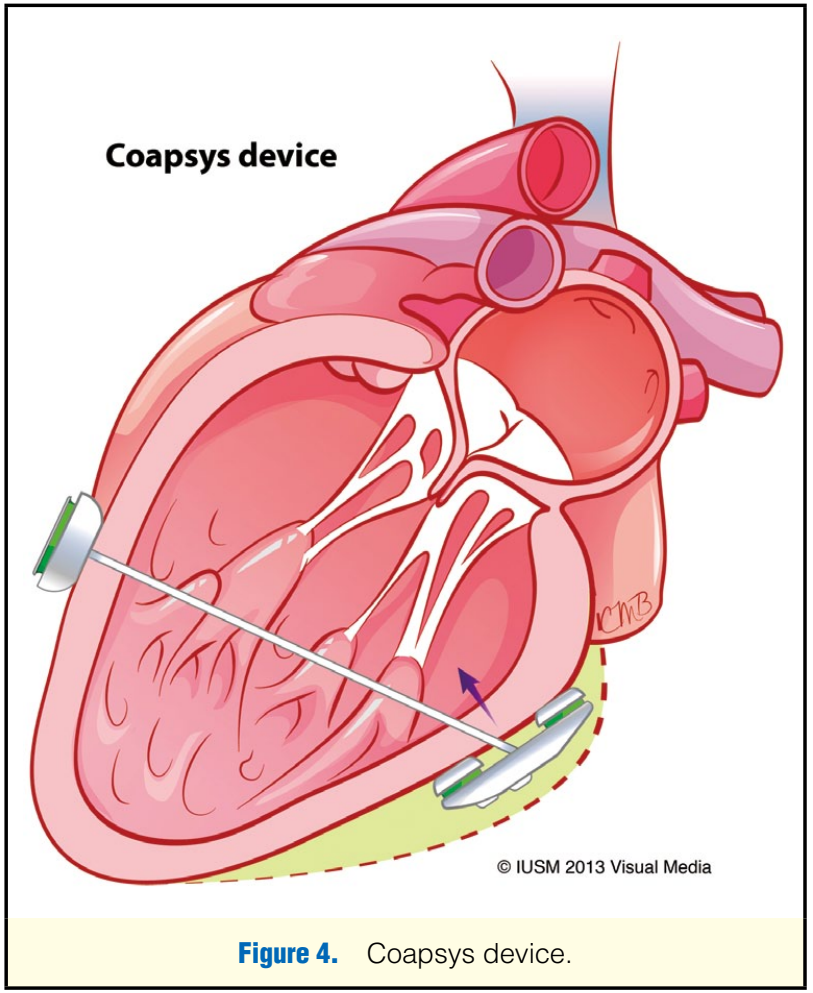

valve must address all facets of the pathology, including the etiology, lesion, and resultant dysfunction. Coronary ischemia should be addressed by revascularization where appropriate. Other efforts can be directed at ventricular remodeling, papillary muscle displacement, chordal tethering, and leaflet restriction. Mitral valve replacement with preservation of the subvalvular apparatus with either a mechanical or biologic prosthesis remains preferable to a suboptimal repair.

Newer approaches to repairing the regurgitant ischemic mitral valve target specific characteristics in the mechanism of the disease. One area is the dilated ventricle. Infarcted and chronically ischemic myocardium leads to left ventricular dilatation. External ventricular restraint devices have been, and continue to be, trialed to combat negative left ventricular remodeling. By restraining ventricular dilatation, these devices attempt to replace the papillary muscles in their pre-remodeling location.

Hung et $\mathrm{al}^{8}$ devised a localized patch with an inflatable epicardial balloon (Figure 2). The patch can be placed posteriorly on the beating heart and the volume in the balloon of the patch can then be adjusted, which moves the ventricular wall and reduces papillary muscle displacement. Leaflet tethering is reduced under echocardiographic guidance. Another external restraint device, the Corcap Cardiac Support Device (Acorn Cardiovascular Inc, St. Paul, MN, USA), has been shown to have a long-term beneficial effect on left ventricular reverse remodeling (Figure 3). ${ }^{9}$ Improving left ventricular reverse remodeling has the potential to limit late failures related to ongoing ventricular dilatation.

A third restraint device, the Coapsys device, treats papillary muscle displacement via inward tension on a transventricular strut anchored epicardially (Figure 4). The epicardial anchors are posterior and anterior pads. The posterior pad has 2 heads that alter the shape of the left ventricle: 1 is at the level of the mitral annulus, and the other is at the level of the posteromedial papillary muscle. The anterior pad is adjustable and can 

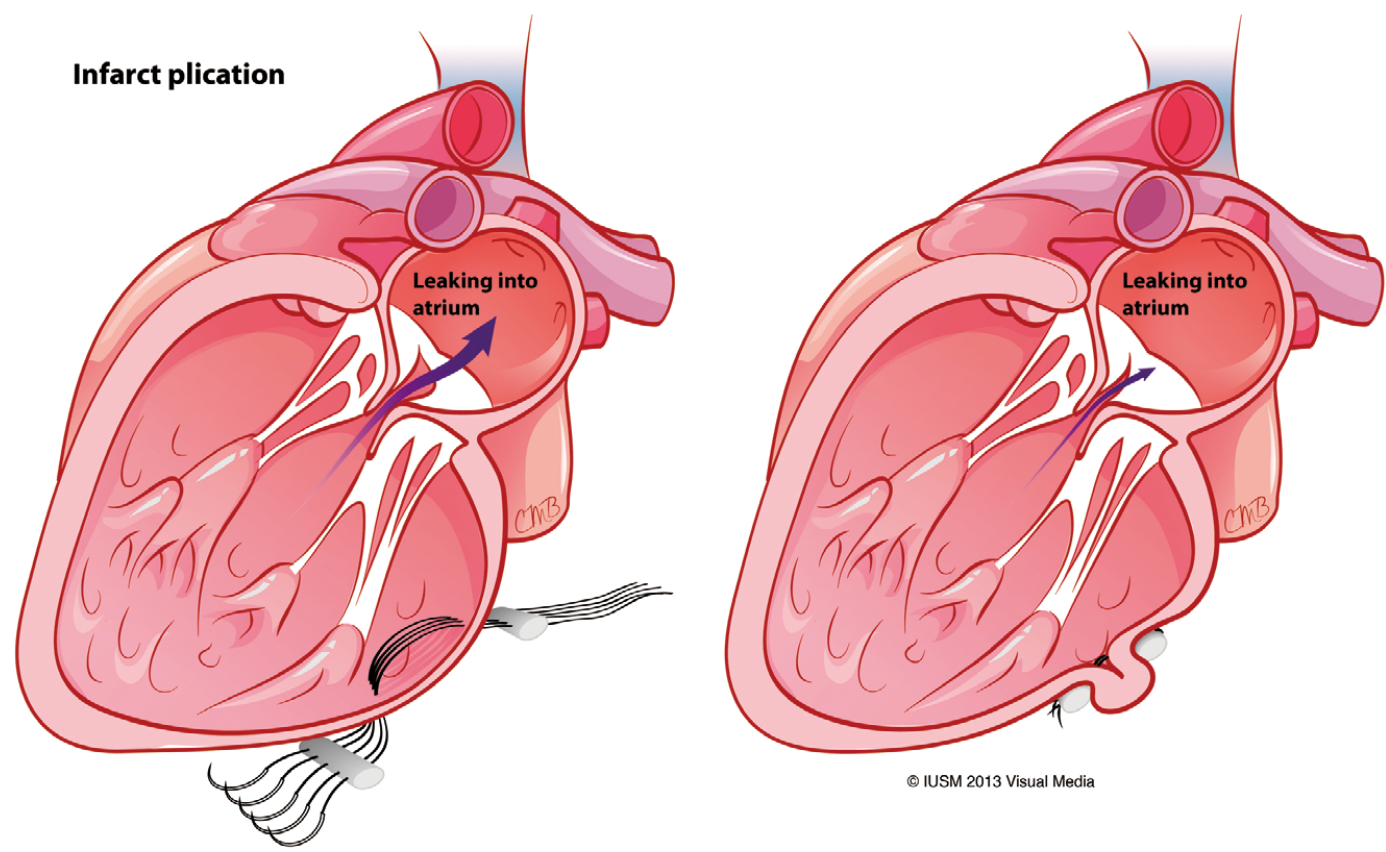

Figure 6. Infarct plication can reduce tethering forces on mitral leaflets.

be fixed after confirming placement of the posterior pad under echocardiographic guidance. A PTFE-coated subvalvular chord connects the pads. Trials of the Coapsys device have demonstrated significantly greater left ventricular reshaping than annuloplasty alone. ${ }^{10}$

Another area of directed focus is the subvalvular apparatus. Subvalvular techniques that address the papillary muscle displacement of chronic IMR include relocation of the posterior papillary muscle, papillary muscle approximation, the papillary muscle sling, and papillary muscle repositioning by infarct plication. ${ }^{2}$ Relocation of the posterior papillary muscle has been performed by connecting it to the mitral annulus close to the right trigone by a suture and then shortening the distance by tying it down, ${ }^{11}$ or, alternatively, connecting the suture from the papillary muscle to the midportion of the anterior annulus and exteriorizing the suture through the aorta so that it can be tied under beating and loaded conditions under echocardiographic guidance. ${ }^{12}$ Papillary muscles have also been approximated via a single mattress suture reinforced with pericardium with some success (Figure 5). ${ }^{13} \mathrm{~A}$ variation of this technique places a Gore-Tex sling around the trabecular bases of both papillary muscles in addition to an undersized annuloplasty ring. ${ }^{14}$ In a highly selected group of patients, plication of an infarct zone (Figure 6) resulted in repositioning of the papillary muscle toward the mitral annulus, reducing chronic IMR. ${ }^{15}$

Although not directly addressing ventricular dilation or papillary muscle displacement, another approach has focused on the role of the chordae tendineae in IMR. The division of strategically located second-order chordae, those attached to the underside of the coapting surface, not including the leading edge or first-order chordae, has been proposed to limit leaflet tethering. Freeing the coapting surfaces of the leaflets from the restriction of secondary chords allows for a greater coapting surface. Limited, early results in an animal model demonstrated no decline in left ventricular function. ${ }^{16} \mathrm{~A}$ clinical trial

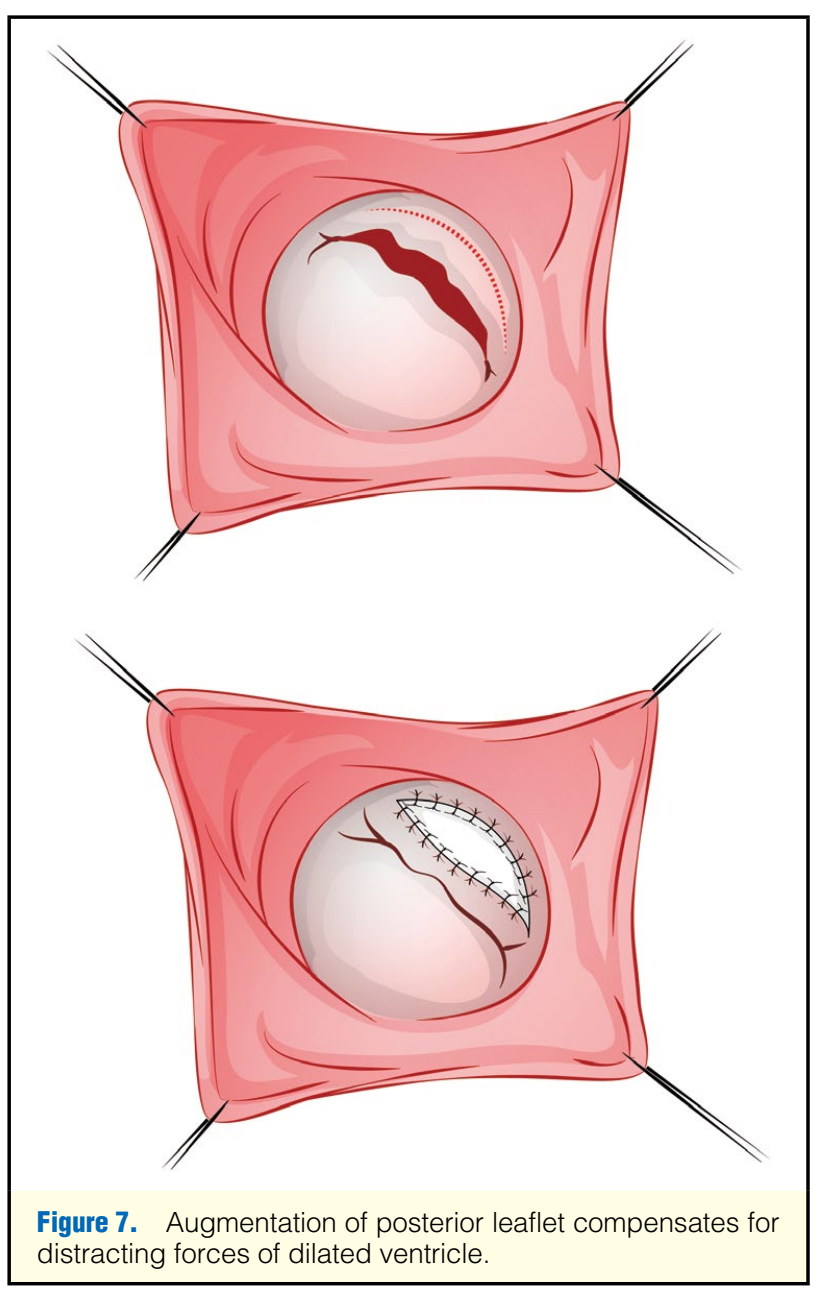


of second-order chordal division and annuloplasty vs. annuloplasty alone demonstrated improved coaptation and a reduction of recurrent IMR. ${ }^{17}$

Surgical therapeutic strategies have also been directed at the leaflets. Kincaid et al introduced anterior leaflet augmentation with a pericardial patch combined with annuloplasty and CABG to address the problem of tethered leaflets in chronic ischemic mitral regurgitation. ${ }^{18}$ Others have advocated patch enlargement of the posterior leaflet (Figure 7). ${ }^{19}$ Another group has recently published on the monocuspidalization of the mitral valve by implantation of a novel bioposterior leaflet, which device imitates a closed posterior mitral leaflet. Implantation of the device restored mitral valvular competence in ischemic IMR because of significant posterior leaflet tethering that not repairable by conventional means. ${ }^{20}$

Restrictive annuloplasty remains applicable in select patients with IMR and limited ventricular dilatation and leaflet tethering. Advances in ring design address pathophysiologic changes of the ischemic ventricle. Early annuloplasty rings were constructed with the mindset that 1 shape fits all. Recognizing that annular and ventricular changes are often asymmetric in IMR, and that the natural mitral annular shape is similar to that of a saddle, newer generations of annular rings have been designed. ${ }^{21}$

\section{Conclusion}

IMR remains a significant clinical problem. The current surgical approach is limited in its ability to address all the relevant aspects of this disease. A more successful approach will incorporate correction of ventricular change, papillary muscle location, and leaflet restriction.

\section{References}

1. Borger M, Alam A, Murphy P, Doenst T, David T. Chronic ischemic mitral regurgitation: Repair, replace, rethink? Ann Thorac Surg 2006; 81: $1153-1161$.

2. Hickey M, Smith L, Muhlbaier L, Harrell F Jr, Hinohara T, Califf R, et al. Current prognosis of ischemic mitral regurgitation: Implications for future management. Circulation 1988; 78: 151-159.

3. Levine R, Schwammenthal E. Ischemic mitral regurgitation on the threshold of a solution: From paradoxes to unifying concepts. Circulation 2005; 112: 745-758.

4. Bouma W, van der Horst I, Wijdh-den Hamer I, Erasmus M, Zijlstra F, Mariani M, et al. Chronic ischaemic mitral regurgitation: Current treatment results and new mechanism based surgical approaches. Eur J Cardiothorac Surg 2010; 37: 170-185.

5. David T, Armstrong S, Sun Z. Left ventricular function after mitral valve surgery. J Heart Valve Dis 1995; 4(Suppl): S175-S180.
6. Tischler MD, Cooper KA, Rowen M, LeWinter MM. Mitral valve replacement versus mitral valve repair: A Doppler and quantitative stress echocardiographic study. Circulation 1994; 89: 132-137.

7. Bach D, Bolling S. Early improvement in congestive heart failure after correction of secondary mitral regurgitation in end-stage cardiomyopathy. Am Heart J 1995; 129: $1165-1170$.

8. Hung J, Guerrero JL, Handschumacher MD, Supple G, Sullivan S, Levine RA. Reverse ventricular remodeling reduces ischemic mitral regurgitation: Echo-guided device application in the beating heart. Circulation 2002; 106: 2594-2600.

9. Starling R, Jessup M, Oh JK, Sabbah HN, Acker MA, Mann DL, et al. Sustained benefits of the Corcap Cardiac Support Device on left ventricular remodeling: Three year follow-up results from the Acorn clinical trial. Ann Thorac Surg 2007; 84: 1236-1242.

10. Grossi EA, Woo YJ, Schwartz CF, Gangahar DM, Subramanian VA, Patel N, et al. Comparison of Coapsys annuloplasty and internal reduction mitral annuloplasty in the randomized treatment of functional ischemic mitral regurgitation: Impact on the left ventricle. J Thorac Cardiovasc Surg 2006; 131: 1095-1098.

11. Kron I, Green G, Cope J. Surgical relocation on the posterior papillary muscle in chronic ischemic mitral regurgitation. Ann Thorac Surg 2002; 74: 600-601.

12. Langer F, Kunihara T, Hell K, Schramm R, Schmidt K, Aicher D, et al. RING plus STRING: Papillary muscle repositioning as an adjunctive repair technique for ischemic mitral regurgitation. J Thorac Cardiovasc Surg 2007; 144: 247-249.

13. Rama A, Praschker L, Barreda E, Gandjbakhch I. Papillary muscle approximation for functional ischemic mitral regurgitation. Ann Thorac Surg 2007; 84: 2130-2131.

14. Hvass U, Tapia M, Baron F, Pouzet B, Shafy A. Papillary muscle sling: A new functional approach to mitral repair in patients with ischemic left ventricular dysfunction and functional mitral regurgitation. Ann Thorac Surg 2003; 75: 809-811.

15. Ramadan R, Al-Attar N, Mohammadi S, Ghostine S, Azmoun A, Therasse A, et al. Left ventricular infarct plication restores mitral function in chronic ischemic mitral regurgitation. $J$ Thorac Cardiovasc Surg 2005; 129: 440-442.

16. Messas E, Pouzet B, Touchot B, Guerrero J, Vlahakes G, Desnos M, et al. Efficacy of chordal cutting to relieve chronic persistent ischemic mitral regurgitation. Circulation 2003; 108(Suppl): II111-II115.

17. Borger M, Murphy P, Alam A, Fazel S, Maganti M, Armstrong S, et al. Initial results of the chordal-cutting operation for ischemic mitral regurgitation. J Thorac Cardiovasc Surg 2007; 133: 1483-1492.

18. Kincaid E, Riley R, Himes M, Hammon J, Kon N. Anterior leaflet augmentation for ischemic mitral regurgitation. Ann Thorac Surg 2004; 78: 564-568.

19. Dobre M, Koul B, Rojer A. Anatomic and physiologic correction of the restricted posterior mitral leaflet motion in chronic ischemic mitral regurgitation. J Thorac Cardiovasc Surg 2000; 120: 409-411.

20. Oertl F, Golczyk K, Pantele S, Danov V, Galinanes M, Beyer M. Mitral valve restoration using the No-React ${ }^{\circledR}$ MitroFix ${ }^{\mathrm{TM}}$ : A novel concept. J Cardiothorac Surg 2012 September 4, doi:10.1186/17498090-7-82 [E-pub ahead of print].

21. Daimon M, Fukuda S, Adams D, McCarthy P, Gillinov M, Carpentier A, et al. Mitral valve repair with Carpentier-McCarthy-Adams IMR ETlogix annuloplasty ring for ischemic mitral regurgitation: Early echocardiographic results from a multi-center study. Circulation 2006; 114: $1588-1593$. 